\title{
Instrument to Streamline the Process of Sending Biological Samples to an Epidemiological Surveillance Center, from a Reference Unit for Infectious and Parasitic Diseases
}

\author{
Evela da Silva Magno ${ }^{1 *}$, Eyde Cristianne Saraiva-Bonatto ${ }^{2}$ and Maria das Graças Gomes Saraiva ${ }^{1,3}$ \\ ${ }^{1}$ Fundação de Medicina Tropical Doutor Heitor Vieira Dourado, Manaus, Amazonas, Brasil \\ ${ }^{2}$ Universidade Federal do Amazonas, Manaus, Amazonas, Brasil \\ ${ }^{3}$ Universidade Nilton Lins, Manaus, Amazonas, Brasil
}

\begin{abstract}
Introduction: The sending of biological materials for specific exams to the Public Health Laboratory of Amazonas (LACEN-AM) depends on documents containing essentia information to guide the performance of the tests, which need to go through a quality process and in most cases the lack of data compromises the streamflow to LACEN-AM.

Objective: Create a checklist for the process of sending samples to the Public Health Laboratory (LACEN-AM).

Methods: The Health Surveillance Guide (GVS), Integrated Manual for Epidemiological Surveillance of botulism, technical notes, reference technicians for information on registration and tests performed at LACEN-AM were consulted and the sequence of activities was also observed. carried out in the notification epidemiology sector, the orientation of the collection of biological material until the sending of the samples.

Results: Between 2017 and 2019, 6 lists were sent to send samples to LACEN-AM, one list for each disease (Paracoccidioidomycosis, Hydatidosis, Severe Acute Respiratory Syndrome, Acute Flaccid Paralysis, Human Rabies and Botulism).

Conclusion: It is necessary to simplify the procedures related to the notification activities for sending biological material samples, improving operationalization, especially for paracocociodiomycosis, hydatidosis and SRAG, diseases that are not included in the GVS, making it difficult to understand the flow of sending biological materials when requested or, in the cases of (PFA), human rabies and botulism which, because they are in the process of monitoring, have their flows difficult to discern. Therefore, disseminating these procedures by means of an instrument can contribute to the improvement and understanding of the technicians who work in epidemiological surveillance, and will certainly speed up their actions, ensuring speed in sending the samples.
\end{abstract}

Keywords: Epidemiology $•$ Cheklist $•$ Operationalization

\section{Introduction}

Parameters and standards of effectiveness and efficiency of health actions, instruments for monitoring and evaluating services, concrete mechanisms for defining agreed goals, as well as quality certification practices have been identified as essential in the processes of integration of care $[1,2]$.

Standardization aims to guarantee the execution of processes always in the same way in order to obtain greater predictability of results. Because it contributes to reducing the variability of production processes, standardization plays an important role in controlling and improving quality in companies [3].

Standardizing processes occurs when the company starts to document its activities, in order to standardize macro and micro processes. That way, regardless of who will perform a specific task, the expected result will always be obtained [4].

Thus, it is possible to constantly manage the quality of activities, bringing improvements and correcting them whenever necessary [4].

*Address for Correspondence: Evela da Silva Magno, Fundação de Medicina Tropical Doutor Heitor Vieira Dourado, Gerência de Epidemiologia.Av, Pedro Teixeira, $n^{0}$ 25, Bairro Dom Pedro, Manaus, Amazonas, Brasil, E-mail: evelamagno_2011@hotmail.com

Copyright: (c) 2020 Silva Magno ED, et al. This is an open-access article distributed under the terms of the Creative Commons Attribution License, which permits unrestricted use, distribution, and reproduction in any medium, provided the original author and source are credited.
The main objective of any process improvement in the health area is to improve the quality of services provided to the patient [5]. Therefore, the objective of this work was to create checklists for the process of sending samples to the Public Health Laboratory (LACEN-AM).

\section{Description of Work Techniques, Methods or Processes}

For a list on paracoccidioidomycosis, a LACEN-AM technician was consulted, who informed the registration of the exam as a search for deep mycoses (Aspergillosis, Histoplasmosis and Paracoccidioidomycosis) and guided the need for clinical history and SUS exam request form, to accompany the delivery of the sample in the laboratory. The LAG was consulted to verify the technique used in the exam and the service practice ordered the sequence of activities on the list.

\section{Hydatidosis}

A consultation with the LACEN-AM technician was carried out, who informed that the hydatidosis form was available electronically for printing and, together with the clinical history and SUS exam request form, are documents that accompany the delivery of the sample at laboratory. The LAG was consulted to verify the technique for performing the exam and the service practice ordered the activities on the list.

\section{Severe Acute Respiratory Syndrome}

(Sars)

Technical note 010/2019-FVS-AM/SUSAM-conduct was consulted in 
Table 1. Diseases not included in the Health Surveillance Guide, (1) paracoccidioidomycosis, (2) hydatidosis and (3) severe acute respiratory syndrome.

\begin{tabular}{|c|c|c|}
\hline List number & Diseases & Orientations \\
\hline \multirow[t]{5}{*}{1} & Paracoccidioidomycosis & () complete a notification/conclusion form of SINAN (annex 1). \\
\hline & & () obtain a SUS exam request form (annex 2)+clinical history, both duly detailed and signed by the requesting physician. \\
\hline & & () guide collection: material: serum (technique used- double radial immunodiffusion). \\
\hline & & () Request GAL:in general clinical data register as ringworm and in research/exams: search for deep mycoses. \\
\hline & & $\begin{array}{l}\text { () attach a copy of the notification+GAL request+list of those sent by the GAL and deliver it to the unit's laboratory to } \\
\text { separate the collected sample and arrange for it to be sent to LACEN-AM. }\end{array}$ \\
\hline \multirow[t]{6}{*}{2} & Hydatidosis & () monitor the status of the exam in the GAL. \\
\hline & & () fill out an epidemiological form for hydatidosis-FIOCRUZ RJ (annex 3). \\
\hline & & () obtain a SUS exam request form (annex 2)+clinical history, both duly detailed and signed by the requesting physician. \\
\hline & & () guide collection: material: serum (technique used-enzyme immunoassay and immunoblot both IgG). \\
\hline & & $\begin{array}{l}\text { () Requisition GAL: general clinical data field disease/disease register as hydatidosis and in research/exams:hydatidosis } \\
\text { serology. }\end{array}$ \\
\hline & & $\begin{array}{l}\text { () attach a copy of the notification+GAL request+list of those sent by the GAL and deliver it to the unit's laboratory to } \\
\text { separate the sample collected and send it to LACEN-AM, which will forward it to FIOCRUZ RJ. }\end{array}$ \\
\hline \multirow[t]{7}{*}{3} & $\begin{array}{l}\text { Severe Acute Respiratory } \\
\text { Syndrome (Sars) }\end{array}$ & () monitor the status of the exam in the GAL. \\
\hline & & () fill in the standardized form for severe acute respiratory syndrome. \\
\hline & & () send the form to the Health District. \\
\hline & & $\begin{array}{l}\text { () inform that the collected material is aspirated nasopharynx (technique used to search for influenza and other respiratory } \\
\text { viruses-RT-PCR in real time and direct immunofluorescence) and that the Ministry of Health recommends the collection of } \\
\text { samples until the 7th day after the beginning symptoms. }\end{array}$ \\
\hline & & $\begin{array}{l}\text { () GAL requisition register: in general clinical data in the field of disease/illness register as influenza/respiratory viruses and } \\
\text { in research/tests: influenza and other respiratory viruses. }\end{array}$ \\
\hline & & $\begin{array}{l}\text { () attach a copy of the notification+requisition+list of forwarded from the GAL and activate LACEN-AM to carry out the } \\
\text { collection. }\end{array}$ \\
\hline & & () monitor the status of the exam in the GAL. \\
\hline
\end{tabular}

Source: Laboratory environment manager (GAL), technical notes and reference technicians LACEN-AM

Table 2. Diseases guided by the Health Surveillance Guide, (4) acute flaccid paralysis, (5) human rabies and (6) botulismo.

\begin{tabular}{|c|c|}
\hline List number Diseases & Orientations \\
\hline PFA & () fill in a standardized form for PFA \\
\hline 4 & () send a form to the Health District. \\
\hline & $\begin{array}{l}\text { () inform that the collected material is made for viral isolation; the ministry of health recommends collecting the sample by the 14th day } \\
\text { after the onset of symptoms. }\end{array}$ \\
\hline & $\begin{array}{l}\text { () GAL requirement register: general clinical data in the field of disease/illness register as acute flaccid paralysis/polio and in research/ } \\
\text { tests: polio/acute flaccid paralysis. }\end{array}$ \\
\hline & $\begin{array}{l}\text { () attach a copy of the notification+GAL's request+GAL's forwarding list and deliver it to the unit's laboratory to separate a collected } \\
\text { sample and still send it to LACEN-AM. }\end{array}$ \\
\hline Human rage & () monitor the exam status in the GAL \\
\hline \multirow{10}{*}{5} & () fill in the standard form for human rabies \\
\hline & () send the form to the Health District. \\
\hline & $\begin{array}{l}\text { () inform the material to be collected: serum (biological test and direct immunofluorescence), cerebrospinal fluid (PCR), saliva (PCR and } \\
\text { biological test) and hair follicle (PCR and complementary sequencing exam). }\end{array}$ \\
\hline & $\begin{array}{l}\text { () GAL requisition register: in general clinical data in the disease/disease field register as human rabies and in research/exams: diagnostic } \\
\text { rage for the serum sample, diagnostic rage (CSF), diagnostic rage (saliva) and diagnostic rage (hair follicle). }\end{array}$ \\
\hline & () attach a copy of the notification+GAL request+GAL forwarding list and, activate LACEN-AM to perform the collection. \\
\hline & () monitor the status of exams in the GAL. \\
\hline & () fill in the standardized form for botulism \\
\hline & () send the form to the Health District. \\
\hline & $\begin{array}{l}\text { () inform the biological material to be collected: blood in sufficient quantity to obtain } 11 \mathrm{ml} \text { of serum and feces to collect at least } 15 \mathrm{~g} \text { or } \mathrm{ml} \\
\text { (at most up to } 7 \text { days after the onset of symptoms) for the detection of botulinum toxin. }\end{array}$ \\
\hline & () GAL requisition register: in general clinical data in the disease/illness field register as botulism and in research/exams: botulism \\
\hline Botulism & $\begin{array}{l}\text { () attach a copy of the notification+GAL request+GAL forwarding list and deliver it to the unit's laboratory to separate the collected sample } \\
\text { and also arrange for it to be sent to LACEN-AM. }\end{array}$ \\
\hline & () monitor the status of exams in the GAL. \\
\hline
\end{tabular}

Source: Health surveillance guide, botulism technical manual, technical notes and GAL.

the case of SARS-AM of March 4, March 19. The technique used to perform the exam was consulted at the GAL and the activities on the list followed the order in which the service was operationalized.
For the list of acute flaccid paralysis, human rabies and botulism - The health surveillance guide and the botulism manual for instruction in the collection of biological material were consulted. The LAG was consulted to 
verify the techniques used to perform the exams and the service's actions served to order the activities on the list.

\section{Results}

Six instruments (checklist) were carried out from 2017 to 2019, one for each disease (Paracoccidioidomycosis, Hydatidosis, Severe Acute Respiratory Syndrome, Acute Flaccid Paralysis, Human Rabies and Botulism) Tables 1 and 2, which simplified the sending of biological material to the Public Health Laboratory of Amazonas (LACEN-AM), even if these exams were occasionally requested.

The product is a resource that establishes the sequence of tasks to be accomplished by organizing the activities for sending a sample of biological material to the Public Health Laboratory.

Regardless if the health professional knows the specific manuals for the illnesses, the technical notes, the corresponding chapter in the Health Surveillance Guide or consulted a laboratory reference technician to clarify any doubts, even so this worker only using the instrument (checklist) ) will be able to explain the techniques that will be used in carrying out the exams, inform the material to be collected and the time in which the collection should be carried out, will even know if the notification uses a specific form or not and when registering a sample in the GAL only using the specific list you will be able to complete the registration, fulfilling the routine of sending biological material, with minimal knowledge of the operationalization of the epidemiological surveillance sector, optimizing the time and speeding up the sending of material.

\section{Conclusion}

Some diseases, despite not occurring in an endemic way or being in the process of monitoring, have the tests carried out at the Public Health Laboratory (LACEN), and a Reference Unit in infectious disease may eventually have this demand even if occasionally to meet, therefore, it is of fundamental importance to know this flow, even if a quick check to the checklist is carried out, preventing an examination from being carried out, to clarify the diagnosis or to monitor possible re-introduction of the disease.

With the diseases not covered in the Health Surveillance Guide, we had great difficulty in obtaining the first information about the exam and if it was performed by the central laboratory (LACEN), so the creation of tools that standardize this task can help in the routine of the professional who it usually deals with other demands in the epidemiological surveillance activity.

It is necessary to simplify the procedures related to the notification activities for sending biological material, to improve operationalization, especially for paracocociodiomycosis, hydatidosis and Severe Acute Respiratory Syndrome (SARS) diseases that are not covered in the Health Surveillance Guide (GVS), making it difficult to understanding of the flow of sending biological materials when requested or of diseases such as Acute Flaccid Paralysis (PFA), human rabies and botulism which, due to being in the process of monitoring, have their flows difficult to discern. Therefore, disseminating these procedures through an instrument can contribute to the improvement and understanding of the technicians who work in epidemiological surveillance, and it will certainly speed up their actions, guaranteeing speed in sending the samples in the diagnostic clarification and in the monitoring of diseases that can be reintroduced.

\section{References}

1. Kodner, Dennis L, and Cor Spreeuwenberg. "Integrated care: meaning, logic, applications and implications: a discussion paper." International Journal of Integrated Care, Netherlands 14 (2002):1-6.

2. Smith, Graeme, and David Clarke. "Assessing the effectiveness of integrated interventions: terminology and approach." Medical Clinics of North America 90 (2006):533-548

3. Teixeira, Priscila Carmem, André Felipe Correa Cervi, Daniel Jugend and Otávio José de Oliveira, et al. "Padronização e melhoria de processos produtivos em empresas: estudo de múltiplos casos." Production 24 (2014):311-321.

4. Felix, Schultz. "Padronização de processos: melhore a produtividade da sua empresa". ERP BomControle 2019.

5. Berlitz, Fernando de Almeida. "Quality control in the clinical laboratory: aligning process improvement, reliability and patient safety." J Bras Patol Med Lab 46 (2010):353-363.

How to cite this article: Evela da Silva Magno, Eyde Cristianne SaraivaBonatto and Maria das Graças Gomes Saraiva. Instrument to Streamline the Process of Sending Biological Samples to an Epidemiological Surveillance Center, from a Reference Unit for Infectious and Parasitic Diseases. Clin Infect Dis 4 (2020) doi: 10.37421/jid.2020.4.120 